\title{
Vertically Aligned and Interconnected Graphene Networks for High Thermal Conductivity of Epoxy Composites with Ultralow Loading
}

Gang Lian, ${ }^{*}{ }^{*}$ Chia-Chi Tuan, ${ }^{\star}$ Liyi Li, ${ }^{*}$ Shilong Jiao, ${ }^{\dagger}$ Qilong Wang, ${ }^{\square}$ Deliang Cui, ${ }^{* \dagger}$ and Ching-Ping Wong, * *

\section{Outline:}

Scheme S1. Optimizing process of parameters to prepare vertically aligned graphene interconnected networks.

Scheme S2. Schematic illustration of preparing double-layer sample.

Scheme S3. Schematic illustration of the single-layer and double-layer modes for thermal diffusivity measurement.

Figure S1. Disordered GO network obtained by liquid-nitrogen freezing method under a GO concentration of $20 \mathrm{mg} \mathrm{mL}^{-1}$.

Figure S2. Optical image of GO foam obtained under a concentration of $30 \mathrm{mg} \mathrm{mL}^{-1}$. Figure S3. SEM images of graphene foam microstructures after flame reduction.

Figure S4. SEM and optical images of thin VAIGN-epoxy composite.

Figure S5. Thermal conductivity of random porous graphene network composites.

Table S1. K and CTE value comparisons at different loading fraction. 


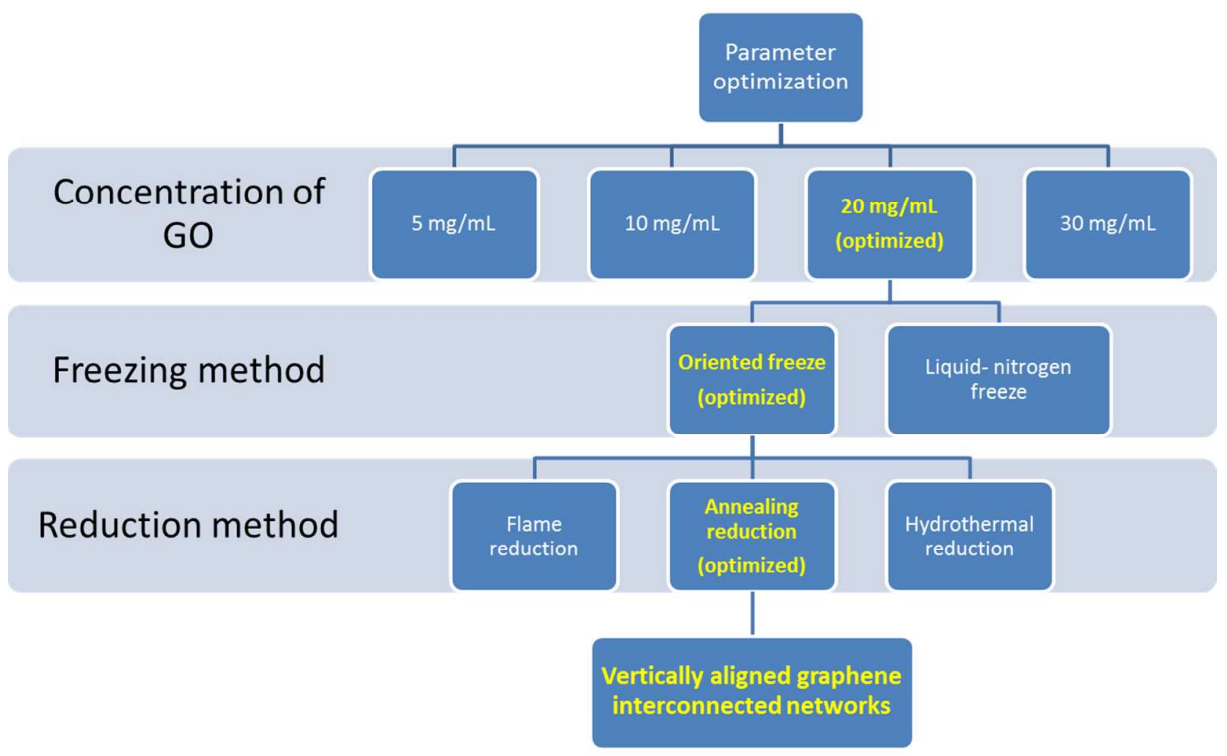

Scheme S1. Optimizing process of parameters to prepare vertically aligned graphene interconnected networks. 


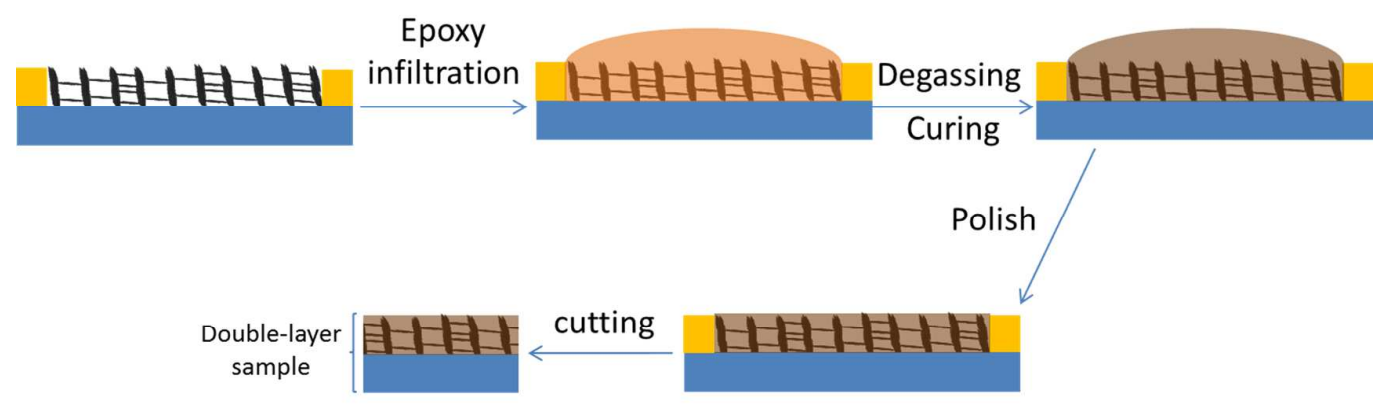

Scheme S2. Schematic illustration of preparing double-layer sample. 
(a)

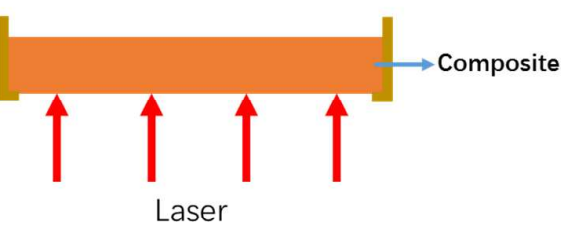

Single-layer mode (b)

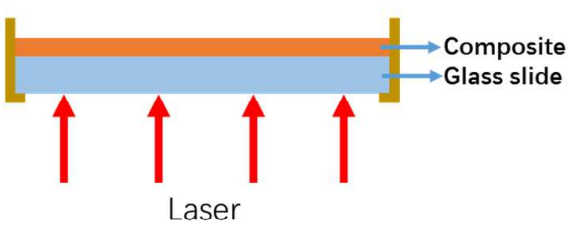

Double-layer mode

Scheme S3. Schematic illustration of the (a) single-layer and (b) double-layer modes for thermal diffusivity measurement. Generally, the single-layer mode is used to test the thermal diffusivity of the composites. However, when the composites are very thin, some substrates are essential to support the composites in preparing the composites process. Then we need to use double-layer mode to test the thermal diffusivity of these composites. Herein, the first layer is the glass slide and the second layer is the thin composite. 


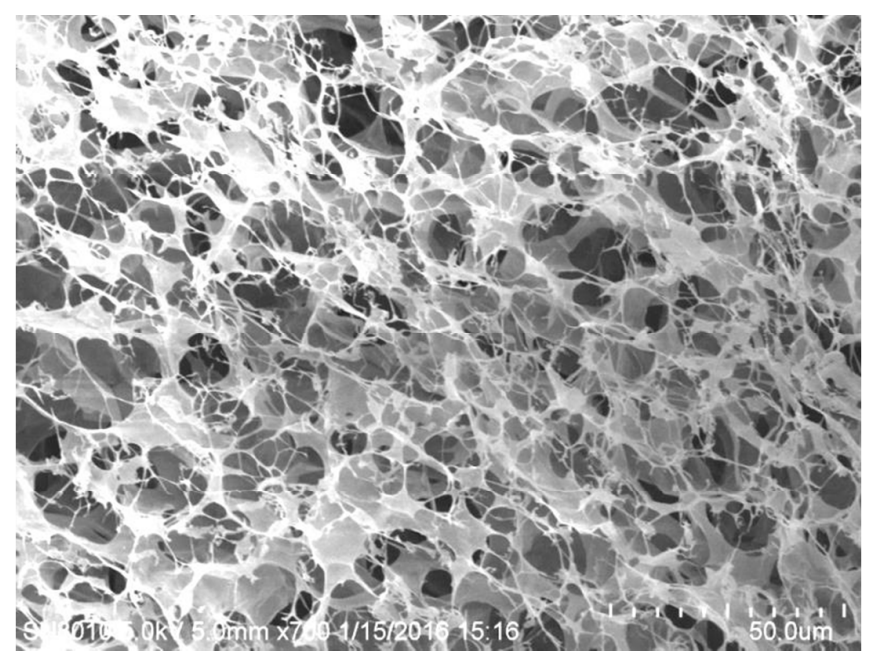

Figure S1. Disordered GO network obtained by liquid-nitrogen freezing method under a $\mathrm{GO}$ concentration of $20 \mathrm{mg} \mathrm{mL}^{-1}$. 


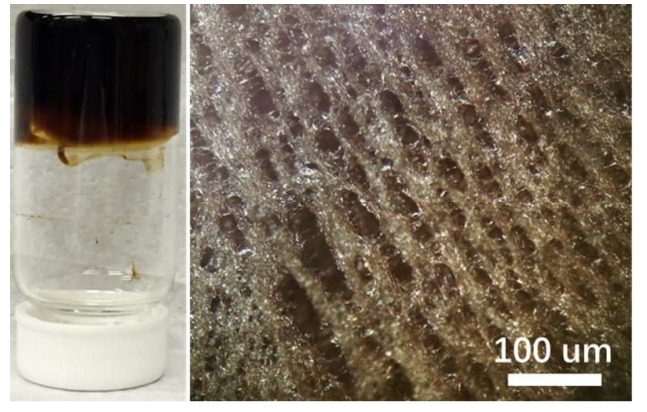

Figure S2. Optical image of GO suspension and foam obtained under a concentration of $30 \mathrm{mg} \mathrm{mL}^{-1}$. 


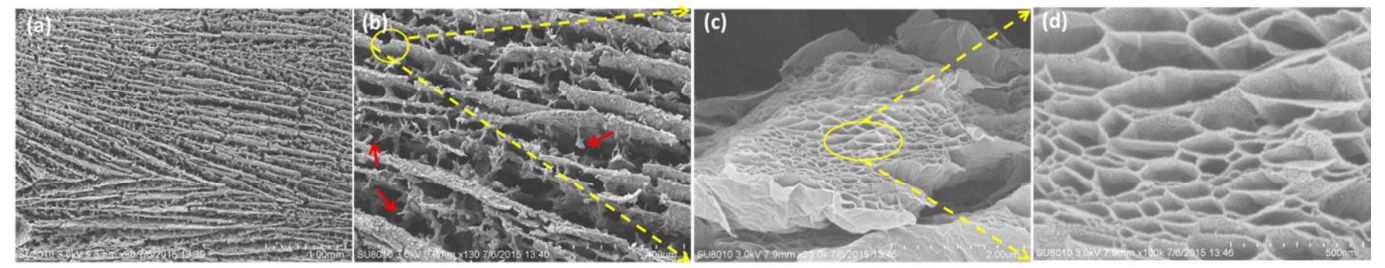

Figure S3. SEM images of graphene foam microstructures after flame reduction. 


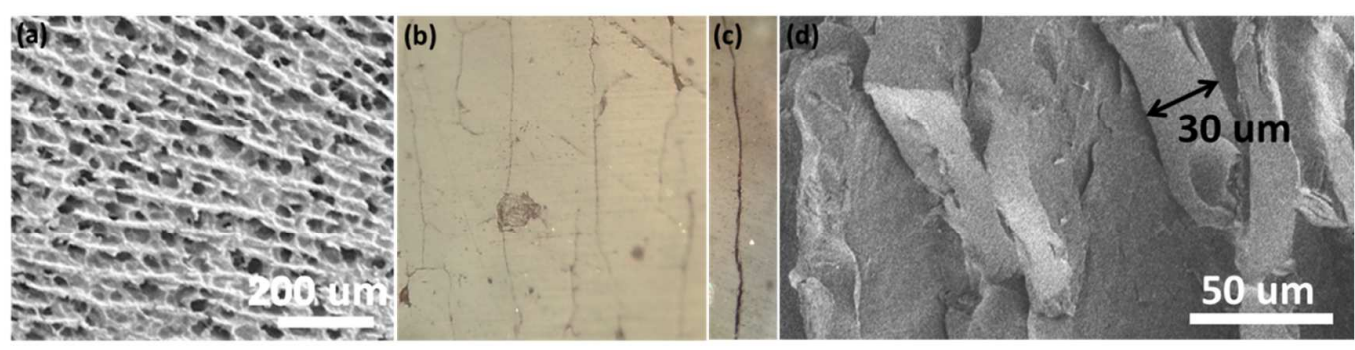

Figure S4. (a) Top-view SEM image of thin VAIGN-epoxy composite (before polishing). When the top surface of VAIGN was completely covered by epoxy resin, it is difficult to observe the integrity of VAIGN. For convenient observation, a composite, not fully infiltrated by epoxy, was prepared. It is shown the vertically aligned microstructures were still preserved in the matrix. (b-c) Top-view optical images of composites with different magnification (after polishing). They clearly present the vertically aligned, long graphene walls in the matrix. (d) Side-view SEM image of the broken composite. The parallel polymer blocks in the composite were confined by the vertically aligned graphene walls, which existed between the blocks.

The thickness of individual polymer block is $\sim 30 \mathrm{um}$, similar with the distance between the adjacent graphene walls in the VAIGN, which also supports the integrity of VAIGN preserved. 


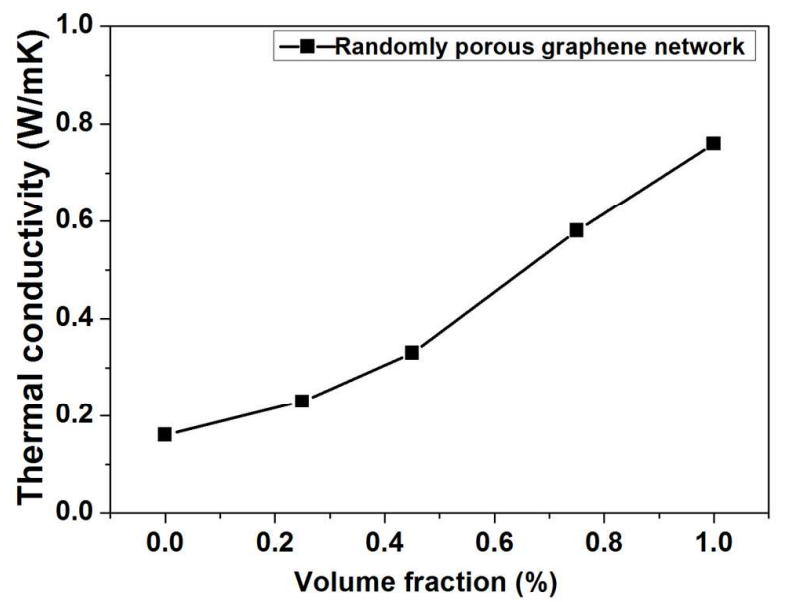

Figure S5. Thermal conductivity of random porous graphene network composites. The random network was obtained by liquid-nitrogen freeze casting, which is an isotropic method. The typical microstructure is shown in Figure S1. 


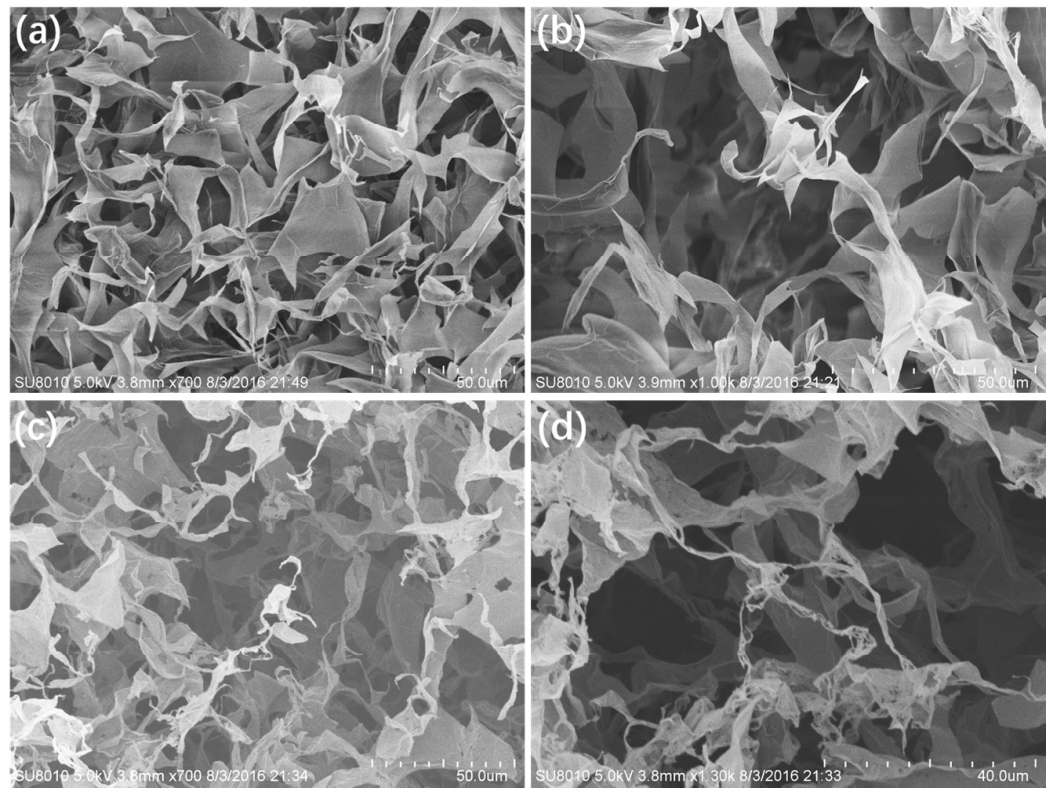

Figure S6. (a-b) SEM images of GO foam obtained under a concentration of $5 \mathrm{mg} \mathrm{g}^{-1}$. (c-d) The corresponding rGO foam after annealing. 
Table S1. K and CTE value comparisons at different loading fraction.

\begin{tabular}{|c|c|c|c|c|c|}
\hline $\begin{array}{c}\text { fraction } \\
(\mathrm{vol} \%)\end{array}$ & $\begin{array}{c}\mathrm{Cp} \\
\left(\mathrm{J} \mathrm{g}^{-1} \mathrm{C}^{-1}\right)\end{array}$ & $\begin{array}{c}\rho \\
\left(\mathrm{g} \mathrm{cm}^{-3}\right)\end{array}$ & $\begin{array}{c}\alpha \\
\left(\mathrm{mm}^{2} / \mathrm{s}\right)\end{array}$ & $\begin{array}{c}\mathrm{K} \\
\left(\mathrm{W} \mathrm{m}^{-1} \mathrm{~K}^{-1}\right)\end{array}$ & $\begin{array}{c}\text { CTE below } \\
\left.\mathrm{T}_{\mathrm{g}}(\mathrm{ppm} \mathrm{K})^{-1}\right)\end{array}$ \\
\hline 0 & 1.19 & 1.24 & 0.1 & 0.16 & 78.9 \\
\hline 0.2 & 1.187 & 1.24 & $0.146 / 0.15 / 0.151$ & $0.215 / 0.221 / 0.223$ & 70.6 \\
\hline 0.45 & 1.185 & 1.25 & $0.304 / 0.324 / 0.311$ & $0.45 / 0.48 / 0.46$ & 64.9 \\
\hline 0.52 & 1.182 & 1.25 & $0.48 / 0.467 / 0.487$ & $0.71 / 0.69 / 0.72$ & 55.8 \\
\hline 0.68 & 1.182 & 1.26 & $0.551 / 0.53 / 0.524$ & $0.82 / 0.79 / 0.78$ & 45.5 \\
\hline 0.75 & 1.17 & 1.26 & $0.65 / 0.62 / 0.739$ & $0.96 / 0.91 / 1.09$ & 41.2 \\
\hline 0.92 & 1.16 & 1.27 & $1.37 / 1.52 / 1.43$ & $2.02 / 2.24 / 2.1$ & 37.4 \\
\hline
\end{tabular}

